Check for updates

Cite this: RSC Adv., 2019, 9, 20029

\title{
Fluorescence sensing of tyrosinase activity based on amine rich carbon dots through direct interaction in a homogeneous system: detection mechanism and application $\uparrow$
}

\author{
Jianzhong Zhang, $t^{\mathrm{a}}$ Yuyuan Chen, $t^{\mathrm{b}}$ Zongfu Zheng, ${ }^{\mathrm{c}}$ Zhenzhen Wang, ${ }^{\mathrm{b}}$ \\ Yanjie Zheng, ${ }^{\mathrm{b}}$ Xinhua Lin ${ }^{\mathrm{b}}$ and Shaohuang Weng $\mathbb{I D}^{* \mathrm{~b}}$
}

\begin{abstract}
As a vital, copper-containing oxidase, tyrosinase (TYR) is useful as a biomarker for the screening of skin diseases. In this paper, a convenient and sensitive homogeneous fluorescence detection platform for the assay of TYR activity without any modified steps is described. Inspired by the fact that carbon dots (CDs) with excellent properties can be obtained through some surface modification, amine rich carbon dots $(\mathrm{N}$-CDs) using a nitrogen doping process were developed as the fluorescent probe for this assay. The effect and the response mechanism of the degree of nitrogen doping in relation to the response of different CDs to the sensing of TYR activity using dopamine (DA) as a substrate were investigated. The DA was oxidized to o-dopaquinone with the catalyzation of TYR and quenched the fluorescence of the $\mathrm{N}-\mathrm{CDs}$ by direct interaction. By using a set concentration of DA and other optimized reaction conditions, the fluorescence intensity of the N-CDs was directly applied to monitor the TYR activity. This assay for TYR activity showed a broad linear range from 0.05 to $6.0 \mathrm{U} \mathrm{mL}^{-1}$ with a detection limit of $0.039 \mathrm{U}$ $\mathrm{mL}^{-1}$. The satisfactory recovery of the sensor for TYR activity in diluted human serum illustrated

a potential clinical application.
\end{abstract}

Received 25th April 2019

Accepted 12th June 2019

DOI: $10.1039 /$ c9ra03098k

rsc.li/rsc-advances

\section{Introduction}

Tyrosinase (TYR), a copper-containing enzyme, is widely distributed in animal tissues and plants. ${ }^{1-3}$ As an oxidase, TYR plays an important role in several physiological reactions, such as melanin synthesis in the body, insect cuticle sclerotization and plant browning reactions. ${ }^{3-5}$ In humans, the TYR activity is crucial because it has the role of being an important antigen for the autoimmunity of vitiligo. ${ }^{6}$ Uncontrolled expression of TYR activity can cause some diseases, such as melasma, age spots and vitiligo. ${ }^{6,7}$ Thus, TYR can act as a biomarker for the screening of skin diseases. In addition, in the food industry, TYR is also an influential index of nutritional value, taste, colour and digestibility of fruits and vegetables. ${ }^{3,8}$ Therefore, the

\footnotetext{
${ }^{a}$ Department of Gastric Surgery, Fujian Medical University Union Hospital, Fuzhou 350001, China

${ }^{b}$ Department of Pharmaceutical Analysis, The Higher Educational Key Laboratory for Nano Biomedical Technology of Fujian Province, School of Pharmacy, Fujian Medical University, Fuzhou 350122, China. E-mail: shweng@fjmu.edu.cn

'476 Hospital of PLA, Fuzhou 350002, China

$\dagger$ Electronic supplementary information (ESI) available: TEM images of N-CDs, lifetime decay of different carbon dots, high-resolution XPS spectrum of N1s, and optimized conditions. See DOI: 10.1039/c9ra03098k

$\ddagger$ Both authors contributed equally to this work.
}

development of an assay for the detection of TYR activity is of great value in clinical diagnosis and in food applications.

Until now, lots of sensing platforms have been used for the detection of TYR activity, such as electrochemical, ${ }^{2,9,10}$ colorimetric $^{11}$ and fluorescence methods. ${ }^{3,12-14}$ Among all the methods, fluorescence methods are attractive because of using them for in situ analysis with the advantages of simplicity, sensitivity, repeatability and specificity. As far as is known, TYR can catalyze catechol derivatives to $o$-quinone using a method based on their diphenolase activity., ${ }^{\mathbf{3} 15,16}$ Several fluorescent sensing strategies have been developed using some special fluorophores or fluorescent nanomaterials as probe based on the electron transfer between probes and $o$-quinone and the resultant quenching behavior. ${ }^{3,15,16}$ The covalent conjugation and homogeneous adsorption are the two main interacting forms for the construction of such quenching behavior.,12-18 Whereas, covalent conjugation of substrates on the surface of quantum dots (QDs), such as carbon dots (CDs), is the most important strategy for TYR detection. ${ }^{12-14,16-18}$ However, most of the proposed methods need laborious modification or preparation process of the fluorescent materials. ${ }^{16-18}$ The additional modification process may increase the cost and complexity of the platform. The development of a new assay platform without any modification, using a homogeneous reaction with simple 
blending procedures for the sensing of TYR activity is still urgently required.

The CDs have received increasing attention in the past few years because of their excellent optical properties. The CDs have been extensively applied in biosensing, imaging and drug delivery. ${ }^{19-21}$ In order to achieve the wide range of applications of CDs, a heteroatom doping process, such as the doping of nitrogen $(\mathrm{N})^{22}$ or fluorine ${ }^{23,24}$ elements, is an effective method. In general, $\mathrm{N}$-doping is a common approach for the exploitation of the novel properties of CDs combined with suitable surface modification. For example, N-doped CDs with special surface functional groups and internal fluorescence can be used as an effective quenching platform for the ratiometric fluorescence sensing of DNA. ${ }^{22,25}$ For the development of a special method in a homogeneous system without any conjugation process, it was noted that modulating the surface property of CDs with different groups was a suitable approach for improving the sensing performance and application scope of CDs. ${ }^{22,25,26}$ Several biosensing platforms constructed from N-doped CDs have been reported, ${ }^{22,26}$ however, the systematic evaluation of the different degrees of $\mathrm{N}$-doping with different surface properties on the performance of the analytical application of CDs is still lacking. Furthermore, for the monitoring of TYR activity in a fluorescent homogeneous system with the investigation of the direct interaction of an enzymatic substrate and CDs as a fluorescence signal is necessary.

In this research, three different CDs with various preparation methods to obtain different degrees of N-doping were synthesized. The three CDs of different degrees of $\mathrm{N}$-doping possessed various contents of amines. Using TYR as a model enzyme, the synthetic investigation of the interaction of TYR and different CDs with various surface functional groups was carried out. Under optimal conditions, N-CDs were prepared from citric acid (CA), glutathione (GSH) and polyethylene polyamines (PEPA) and the most sensitive fluorescence quenching response to TYR was obtained with dopamine (DA) as the substrate. Thus, an easy and effective fluorescence method to detect TYR activity using N-CDs as a probe was developed. This method based on $\mathrm{N}$-CDs was also applied to accurately, and conveniently, test the TYR activity in diluted serum, suggesting it could be used for clinical applications in the future.

\section{Experimental section}

\section{Chemicals and materials}

Citric acid and PEPA were purchased from the Aladdin Industrial Corporation (Shanghai, China). Tyrosinase (TYR) from mushrooms, DA, GSH, ascorbic acid (AA), glucose oxidase (GOD), alkaline phosphatase (ALP), glycine (Gly), lysozyme, trypsin and catalase were supplied by Sigma-Aldrich. Cysteine, acetone, sodium chloride, sodium dihydrogen phosphate dodecahydrate $\left(\mathrm{Na}_{2} \mathrm{HPO}_{4} \cdot 12 \mathrm{H}_{2} \mathrm{O}\right)$, sodium phosphate monobasic dihydrate $\left(\mathrm{NaH}_{2} \mathrm{PO}_{4} \cdot 2 \mathrm{H}_{2} \mathrm{O}\right)$ and glucose were obtained from Sinopharm Chemical Reagent Co., Ltd. All aqueous solutions used in this work were prepared in ultrapure water obtained from a Millipore system. All analytical grade reagents were used without any purification.

\section{Characterizations}

Fluorescence spectra were recorded on a Cary Eclipse fluorescence spectrophotometer (Agilent Technologies, USA). The ultraviolet-visible (UV-vis) absorption spectra were measured on a UV-2450 UV-vis spectrophotometer (Shimadzu Corporation, Japan). Fourier transform infrared spectroscopy (FTIR) was carried out using a NICOLET iS50 FTIR spectrometer (ThermoFisher Scientific, USA). The X-ray photoelectron spectroscopy (XPS) was carried out using a ESCALAB 250Xi (ThermoFisher Scientific, USA) and the transmission electron microscopy (TEM) images were obtained using a FEI Talos F200S (ThermoFisher Scientific, USA).

\section{Preparation of carbon dots (CDs) with different degrees of nitrogen doping}

Amine rich doped carbon dots (N-CDs) were prepared using the following method. Briefly, the mixture containing CA $(2.0 \mathrm{~g})$ and GSH $(0.6 \mathrm{~g})$ were placed in an oil bath and heated at $140{ }^{\circ} \mathrm{C}$ until the CA and GSH were completely melted and the color changed from colorless to tan. Then, $10 \mathrm{~mL}$ of PEPA was added to the molten mixture and allowed to react at $200{ }^{\circ} \mathrm{C}$ for $1 \mathrm{~h}$. The resultant mixture was then cooled to room temperature and then dissolved with acetone. The precipitate was obtained after the centrifugation at $6000 \mathrm{rpm}$ for $10 \mathrm{~min}$ and then dissolved in ultrapure water. Finally, the dark solution was dialyzed against a 2000 mesh dialysis bag for $72 \mathrm{~h}$.

For comparison, two other types of CDs with a lower content of $\mathrm{N}$ were also prepared. Pure CDs without $\mathrm{N}$ doping fabricated from only CA were designated as $\mathrm{C}-1$. The $\mathrm{N}$ doped CDs prepared from CA and GSH were designated as $\mathrm{C}-2$. For the preparation of $\mathrm{C}-1,2.0 \mathrm{~g}$ of CA was placed in a beaker and heated at $200{ }^{\circ} \mathrm{C}$ for $30 \mathrm{~min}$. Then, the melted product was dispersed in $100 \mathrm{~mL}$ water with stirring and used as the $\mathrm{C}-1$ stock solution.

For the preparation of $\mathrm{C}-2$, the mixture of $2.0 \mathrm{~g}$ of CA and $0.6 \mathrm{~g}$ GSH were heated at $140{ }^{\circ} \mathrm{C}$ until the mixture was completely melted. Then, the temperature of the melted solution was further increased to $200{ }^{\circ} \mathrm{C}$ for $1 \mathrm{~h}$. The resultant product was cooled to room temperature and then dissolved with $30 \mathrm{~mL}$ of ultrapure water. Finally, the solution was dialyzed and collected after $72 \mathrm{~h}$ dialysis using a 2000 mesh dialysis bag. After dialysis, the solution was diluted to a concentration of 340 $\mu \mathrm{g} \mathrm{mL}^{-1}$.

\section{Assay for the detection of TYR activity}

The N-CDs were diluted with $10 \mathrm{mM}$ of phosphate buffered saline (PBS, $\mathrm{pH}$ 6.6) to a final concentration of $28 \mu \mathrm{g} \mathrm{mL} \mathrm{m}^{-1}$. In a reaction solution (total of $200 \mu \mathrm{L}$ ), the fresh mixture containing $15 \mu \mathrm{L}$ of $\mathrm{N}-\mathrm{CDs}, 20 \mu \mathrm{L}$ of $10 \mathrm{mM}$ DA and a certain concentration of TYR ranging from 0 to $20 \mathrm{U} \mathrm{mL}^{-1}$ were prepared and then used to determine the TYR activity. After incubation at $37^{\circ} \mathrm{C}$ for $2 \mathrm{~h}$, the emission fluorescence spectrum was measured at an excitation wavelength of $350 \mathrm{~nm}$ in the range of 360-600 $\mathrm{nm}$. For the evaluation of the selectivity of the TYR assay, several biological substances including glucose (100 
$\mu \mathrm{M})$, Gly $(10 \mu \mathrm{M})$, cysteine $(10 \mu \mathrm{M})$, AA $(10 \mu \mathrm{M})$, bovine serum albumin (BSA, $\left.0.01 \mathrm{mg} \mathrm{mL}^{-1}\right)$, ALP $\left(5 \mathrm{U} \mathrm{mL}^{-1}\right)$, lysozyme $(5 \mathrm{U}$ $\left.\mathrm{mL}^{-1}\right)$, trypsin $\left(5 \mathrm{U} \mathrm{mL}^{-1}\right)$, catalase $\left(5 \mathrm{U} \mathrm{mL}^{-1}\right)$, and $\mathrm{GOD}(5 \mathrm{U}$ $\mathrm{mL}^{-1}$ ) were selected and determined using the same procedure used for the detection of TYR activity. The excitation and emission slit widths in the fluorescence measurement were $5 \mathrm{~nm}$. The TYR activity was obtained using the difference of the fluorescence response intensity. In this determination procedure, $\Delta F$ was defined as $F_{0}-F$, where $F_{0}$ and $F$ were the fluorescence intensity of emission peak centered at $450 \mathrm{~nm}$ in the absence and presence of TYR, respectively.

\section{The determination of TYR activity in serum}

The human serum, obtained from healthy volunteers with informed consent was approved by the Ethics Committee at the Fujian Medical University Union Hospital (Fuzhou, China). The human serum was used to investigate the possibility of the using the assay for TYR activity in humans. The human serum was centrifuged at $7000 \mathrm{rpm}$ for $10 \mathrm{~min}$ to remove any existing protein. Then the serum was diluted to $1 \%$ with $10 \mathrm{mM}$ PBS (pH 6.6) solution and treated as real samples. ${ }^{11,17}$ Then the recovery of this assay, using the addition of different concentrations of TYR, was investigated using the same procedure shown in the section on "Assay for the detection of TYR activity".

\section{Results and discussion}

\section{The reaction system of TYR, DA and N-CDs}

The optical properties of the N-CDs are shown in Fig. 1. There were two absorption bands centered at $240 \mathrm{~nm}$ and $350 \mathrm{~nm}$ which were ascribed to the $\pi-\pi^{*}$ transition of $\mathrm{C}=\mathrm{C}$ and $\mathrm{n}-\pi^{*}$ transition of $\mathrm{C}=\mathrm{O}$ in the internal aromatic ring structure of CDs, respectively. ${ }^{22}$ Furthermore, the fluorescence emission spectrum of the N-CDs showed a maximum wavelength
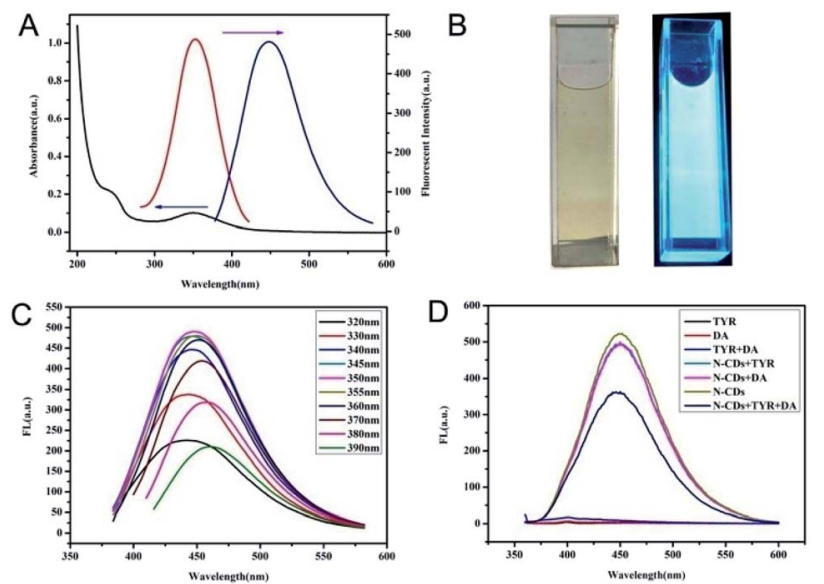

Fig. 1 The UV-vis and fluorescence spectra (A) and images of the aqueous dispersion in daylight (left) and under UV excitation (right) (B). (C) The emission spectra of $\mathrm{N}$-CDs under various excitation wavelengths. (D) Different emission spectra of pure TYR, pure DA, a mixture of TYR and DA, pure N-CDs, a mixture of $\mathrm{N}-\mathrm{CD}$ s and TYR, a mixture of $\mathrm{N}-\mathrm{CDs}$ and DA, and N-CDs in a mixture of TYR and DA with the same excitation wavelength at $350 \mathrm{~nm}$. centered at $450 \mathrm{~nm}$ with $360 \mathrm{~nm}$ excitation. The resultant N-CD solution showed a yellow appearance under daylight and a blue fluorescence under UV light, as shown in the inset of Fig. 1B. The excitation independent luminescence property of N-CDs is shown in Fig. 1C. The morphology of the N-CDs was further investigated using TEM. Fig. S1 (ESI $\dagger$ ) showed that the N-CDs were regular, monodispersed and spherical. The size distribution of the CDs were in the range of 1-4 $\mathrm{nm}$ with an average diameter of $2.5 \mathrm{~nm}$. The high resolution TEM image showed an interplanar spacing of $0.23 \mathrm{~nm}$, which corresponded to the (100) lattice fringe of graphitic carbon. ${ }^{27}$

Using the N-CDs as an effective and cheap probe, the feasibility of using the changed fluorescence of N-CDs for the detection of TYR activity was investigated. The reaction system containing DA as substrate was applied. As shown in Fig. 1D, the characteristic fluorescence of N-CDs centered at $450 \mathrm{~nm}$ was stable in the solutions containing TYR or DA. When TYR and DA were simultaneously mixed with the N-CDs, the sensitive fluorescence quenching by the $\mathrm{N}$-CDs, suggested that the measurement of the degree of fluorescence quenching of the $\mathrm{N}$ CDs could be applied to the quantification of TYR activity. These results laid the foundation for the accurate and sensitive determination of TYR activity using this detection system constructed from $\mathrm{N}$-CDs.

\section{Interaction between TYR, DA, and N-CDs}

It is known that DA can be catalyzed by TYR to form $o$-dopaquinone, one of $o$-quinones, which has been proved to be an efficient fluorescence quencher for fluorescent nanomaterials such as noble metals nanoclusters. ${ }^{3}$ In this work, the electronrich $o$-dopaquinone formed may adsorb on the surface of the $\mathrm{N}$-CDs and shuttle the transfer of electrons between the conduction band and valence band of the N-CDs. The $o$-dopaquinone formed and its adsorption behavior could quench the fluorescence intensity of the N-CDs. In addition, it was reported that quinones generated from TYR can conjugate to amines through Michael-type adduct linkages and/or Schiff bases, ${ }^{28}$ which promoted the interaction of QDs and quinones. For an explanation of the mechanism, the quenching behaviors of three different CDs (N-CDs, C-2 and C-1) with different degrees of $\mathrm{N}$ doping and different surface properties, were tested under different conditions and the results obtained are compared in Fig. 2. The TYR generated $o$-dopaquinone from DA quenched
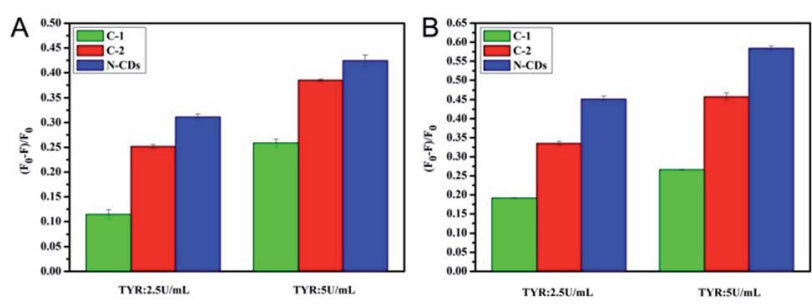

Fig. 2 The different quenching responses of the three types of CDs with $1 \mathrm{mM}$ DA and TYR $\left(2.5 \mathrm{U} \mathrm{mL}^{-1}\right.$ or $\left.5 \mathrm{U} \mathrm{mL}^{-1}\right)$. The original content (A) and original fluorescence intensity (B) of the three types of CDs were controlled to be the same. 
the three CDs, which confirmed the fact that the quinone was an efficient quencher. The decay lifetimes of the three CDs with and without TYR were measured, and the results are shown in Fig. S2 and Table S1 (ESI). $\dagger$ The results showed that the fluorescence lifetime of the three CDs almost remained the same in the sensing system containing TYR or no TYR, proving that the CDs showed a static quenching process with the $o$-dopaquinone formed. ${ }^{29}$ The static quenching process of the CDs occurred because of the formation of some complexes on the CDs because of the adsorption effect of the $o$-dopaquinone as the quenching molecule. ${ }^{29}$

The results in Fig. 2 also show that the quenching efficiency (QE) of the CDs gradually increased with the improved degree of $\mathrm{N}$ doping. This result suggested that the doping effect of $\mathrm{N}$ in the CDs contributed to the sensing performance of the detection of TYR using CDs as the signal. To understand the influence of the doping effect of $\mathrm{N}$ on the mechanism of the different degrees of quenching, XPS and FTIR were carried out to determine the elemental analysis and functional groups of the three CDs. As shown in Fig. 3A, the contents of $\mathrm{N}$ in the N-CDs, C-2 and C-2 were $16.94 \%$, $8.71 \%$ and $0 \%$, respectively. These results confirmed that different reagent sources used in the preparation caused the variable degrees of doping of $\mathrm{N}$ in the CDs. In addition, the N1s XPS spectrum of N-CDs was deconvoluted into two peaks at $398.7 \mathrm{eV}$ and $400 \mathrm{eV}$, which was consistent with the pyridinic $\mathrm{C}-\mathrm{N}-\mathrm{C}$ and graphitic $\mathrm{N}-\mathrm{C}_{3}\left(-\mathrm{NH}_{2}\right)$ (Fig. S3A, ESI $\left.\dagger\right) .{ }^{30}$ The deconvoluted N1s XPS spectrum of C-2 consisted of two peaks at $399.6 \mathrm{eV}$ and $400.3 \mathrm{eV}$, which corresponded to pyrrolic $\mathrm{N}$ and graphitic $\mathrm{N}-\mathrm{C}_{3}\left(-\mathrm{NH}_{2}\right)$ (Fig. S3B, ESI $\dagger$ ). The high resolution of N1s of the N-CDs and C-2 confirmed the presence of $-\mathrm{NH}_{2}$. Using the integral area of graphitic $\mathrm{N}-\mathrm{C}_{3}$, the content of $-\mathrm{NH}_{2}$ in $\mathrm{N}-\mathrm{CDs}$ and $\mathrm{C}-2$ could be calculated to be $7.81 \%$ and $4.36 \%$, respectively. These results suggested that increasing the degree of $\mathrm{N}$ doping would input more $-\mathrm{NH}_{2}$, which could promote the adsorption and reaction of $o$ dopaquinone on the CDs to form a complex. Next, the improved quenching response of the N-CDs was obtained and compared with another two CDs in the detection process of TYR using DA as the substrate. The FTIR spectra of the N-CDs, C-2 and C-1 (Fig. 3B) showed mutual absorption peaks at approximately 3430, 2945 and $1710 \mathrm{~cm}^{-1}$ which were ascribed to the stretching vibrations of $\mathrm{O}-$ $\mathrm{H}, \mathrm{C}-\mathrm{H}$ and $\mathrm{C}=\mathrm{O}$, respectively. Furthermore, the FTIR of the NCDs also showed the obvious vibration peaks of the $\mathrm{N}-\mathrm{H}$ bending vibration at $1556 \mathrm{~cm}^{-1}$, the $\mathrm{C}-\mathrm{NH}-\mathrm{C}$ stretching vibration at $1125 \mathrm{~cm}^{-1}$ and the $-\mathrm{NH}_{2}$ stretching vibration at $3282 \mathrm{~cm}^{-1}$, respectively. In contrast, the FTIR band of the vibration absorption corresponding to $-\mathrm{NH}_{2}$ in $\mathrm{C}-2$ or $\mathrm{C}-1$ was weaker, which was in
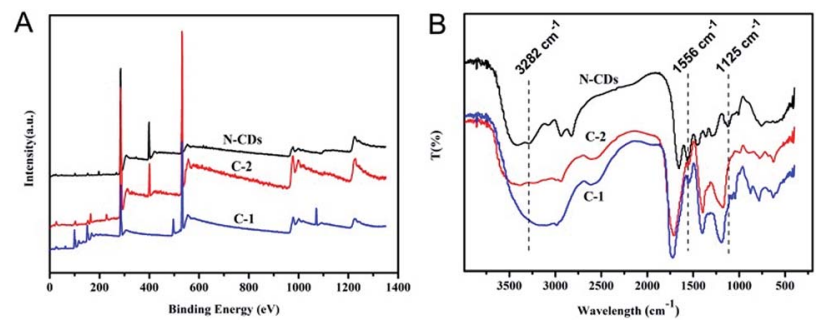

Fig. 3 XPS (A) and FTIR (B) spectra of N-CDs, C-2 and C-1. agreement with the XPS result. The previous results illustrated that the doping process of $\mathrm{N}$ can improve the content of $-\mathrm{NH}_{2}$ on the surface of the CDs. Using DA as a substrate, the increase of $-\mathrm{NH}_{2}$ promoted the adsorption and interaction between $-\mathrm{NH}_{2}$ and TYR generated $o$-dopaquinone, which effectively enhanced the quenching of the fluorescence of the CDs. Thus, the total quenching mechanism of CDs in these conditions was that $o$ dopaquinone would absorb on all kinds of CDs to form some complexes, whereas the existence of $-\mathrm{NH}_{2}$ on the surface of the CDs increased the interaction effect between the CDs and $o$-dopaquinone with the conjugation of quinones and amine, ${ }^{3,28}$ as shown in Scheme 1. This behavior confirmed the feasibility of using the CDs with surface rich $-\mathrm{NH}_{2}$ and DA as a substrate for detection of the TYR activity with improved performance. Thus, the preparation of CDs with special surface modification was a significant factor for the analytical performance.

\section{Optimization of the detection assay for TYR}

To obtain a better detection performance for TYR, several related factors that may affect the response of the N-CDs were optimized prior to the application of the detection system, such as $\mathrm{pH}$, reaction time, temperature and the concentration of DA.

Enzyme activities are mostly influenced by $\mathrm{pH}$. The effect of different $\mathrm{pHs}$ on the TYR activity was investigated first. The NCDs, TYR and DA were mixed in $10 \mathrm{mM}$ PBS at $\mathrm{pH}$ values of $5.0,5.5,6.0,6.3,6.6,7.0$ or 7.4. The fluorescence QE of the $\mathrm{N}$ CDs was obtained using the fluorescence intensity ratio $\left[\left(F_{0}-\right.\right.$ $\left.F) / F_{0}\right]$. The value of $\mathrm{QE}$ increased with the increase of $\mathrm{pH}$ and reached a high value when the $\mathrm{pH}$ was higher than 6.6 (Fig. S4A, ESI $\dagger$ ). Considering the self-polymerization of DA to polydopamine,$^{31-33}$ the quenching effect of pure DA on N-CDs at neutral conditions was also investigated and compared. As shown in Fig. S4B (ESI $\dagger$ ), the fluorescence of the N-CDs was hardly quenched at a pH of 6.6 with the addition of DA. However, the fluorescence of the N-CDs decreased remarkably at $\mathrm{pH} 7.0$ and $\mathrm{pH} 7.4$ (Fig. S4B, ESI $\dagger$ ). The results indicated that alkaline

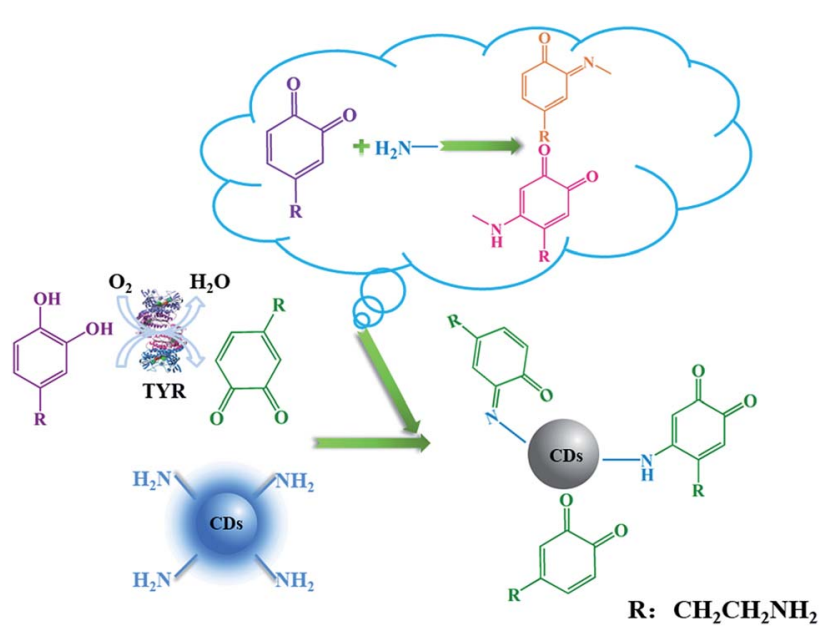

Scheme 1 An illustration of the enzymatic reactions of TYR towards DA and the possible reaction between the CDs and o-dopaquinone to induce the quenching behavior of the CDs for monitoring the TYR activity. 
conditions would confuse the response signal of the detection system for accurate monitoring of TYR. Thus, from the overall consideration of the effect of $\mathrm{pH}$ on the $\mathrm{QE}$ and the stability of DA, in order to minimize the influence of possible oxidation of DA, a pH of 6.6 was chosen as the appropriate one for TYR activity measurement in this assay.

The reaction time and temperature were also optimized for the detection of TYR activity. In general, the reaction time and temperature were interrelated with each other for the evaluation of enzyme activity, in the same way that TYR is in this work. Considering the real application conditions of enzymes and physiological conditions, temperatures of $25{ }^{\circ} \mathrm{C}, 30^{\circ} \mathrm{C}$, and $37^{\circ} \mathrm{C}$ were mainly investigated. Fig. S5A (ESI $\dagger$ ) shows the kinetic reaction processes for TYR activity at $25{ }^{\circ} \mathrm{C}, 30{ }^{\circ} \mathrm{C}$, and $37{ }^{\circ} \mathrm{C}$. With the increase of the reaction period, the $\mathrm{QE}$ was raised at all of the three temperatures. Under the three reaction temperatures, quenching of the N-CDs occurred quickly for the first $60 \mathrm{~min}$, and then gradually reached the maximum degrees of quenching in 120 min. Furthermore, the QE of N-CDs was best at $37{ }^{\circ} \mathrm{C}$. When the reaction time was fixed at $120 \mathrm{~min}$, the fluorescence QE of the N-CDs for the detection of TYR activity was the highest at $37^{\circ} \mathrm{C}$, as shown in Fig. S5B (ESI $\dagger$ ). Therefore, $37{ }^{\circ} \mathrm{C}$ and $120 \mathrm{~min}$ were adopted as the optimal temperature and incubation time, respectively for this assay.

In order to enable the TYR to fully exert its catalytic activity, it was necessary to ensure that there was sufficient DA at a suitable concentration. By varying the concentrations of DA from $0 \mathrm{mM}$ to $2.0 \mathrm{mM}$, the N-CDs were gradually quenched with the increased concentrations of DA in the presence of $20 \mathrm{U} \mathrm{mL}^{-1}$ TYR (Fig. S6A, ESI $\dagger$ ). The quenched fluorescence reached a plateau after 1.0 mM DA was added (Fig. S6B, ESI $\dagger$ ). Thus, 1.0 mM DA was selected as the optimal concentration for TYR detection in this experiment.

\section{Performance of the assay for TYR activity}

Under the optimal reaction conditions, the fluorescence spectra of mixtures containing N-CDs, DA and various concentrations of TYR were monitored at $350 \mathrm{~nm}$ excitation. It was clear from the results shown in Fig. 4A that with the increase of TYR, the fluorescence intensity of N-CDs at about $450 \mathrm{~nm}$ decreased gradually, indicating that the reduced fluorescence was closely dependent on the concentration of TYR. The results in Fig. 4B showed that the value of $\Delta F\left(\Delta F=F_{0}-F\right)$ increased with the increasing concentration of TYR until a plateau was achieved. A good linear relationship between the value of $\Delta F$ and TYR activity could be obtained with TYR concentrations ranging from $0.05-6.0 \mathrm{U} \mathrm{mL}^{-1}$ with the fitting equation of $y=34.30 C_{\mathrm{TYR}}$ [U mL $\left.{ }^{-1}\right]+12.2573$ with $R^{2}=0.9938$ (Fig. 4C). The limit of detection for TYR activity based on a $3 \sigma /$ slope was calculated to be $0.039 \mathrm{U} \mathrm{mL}^{-1}$. A comparison of this method with other previously reported fluorescence assays for TYR activity is shown in Table S2 (ESI $\dagger$ ). The proposed method showed a broad linear range and a comparable detection limit compared to results of other reported fluorescence methods, ${ }^{\mathbf{1 3 , 1 4 , 1 6 , 1 7 , 3 4 , 3 5}}$ whereas the proposed method exhibited the characteristics of a simple mixing procedure without any modification.
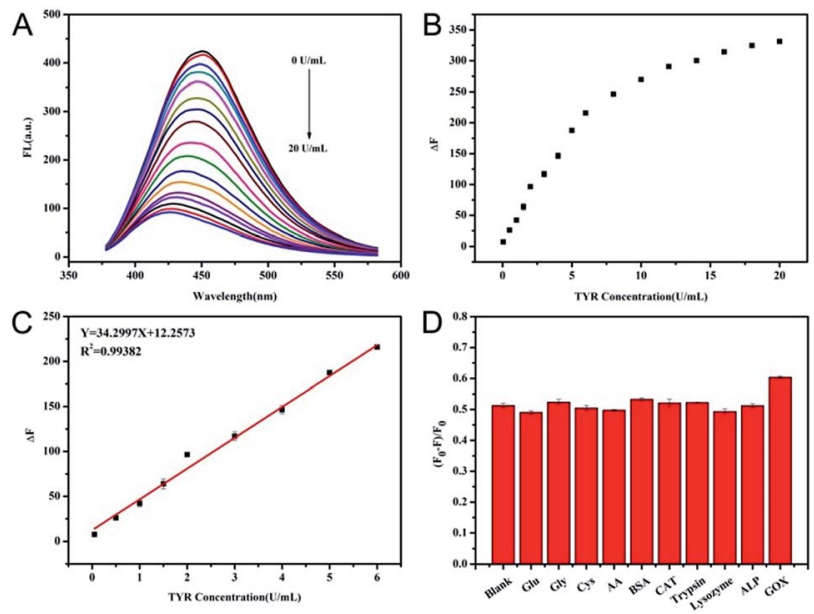

Fig. 4 Fluorescence spectra $(A)$ and the relationship of the degree of quenching (B) of $\mathrm{N}$-CDs in the sensing system with different concentrations of TYR. (C) Linear relationship of $\Delta F$ versus concentrations of TYR, and (D) the specificity of the sensing system for the detection of TYR and the effect of common interferents using the same operating procedure.

In order to assess the anti-interfering ability of the detection assay for TYR activity, some other enzymes or biospecies were chosen to evaluate the possible variations in fluorescence of the $\mathrm{N}-\mathrm{CD}$ using this method. As shown in Fig. 4D, after the ordinary interferents including glucose, Gly, cysteine, AA, BSA, catalase, trypsin, lysozyme, ALP and GOD were added separately into the detection system in the presence of DA, the QE of the NCDs were almost the same in comparison to the unadulterated monitoring of TYR, suggesting that there was a high selectivity with this method for TYR detection.

\section{Determination of TYR activity in serum}

In order to verify the reliability and applicability of the assay for TYR activity in serum, seven human serum samples were spiked with different known concentrations of TYR and were then investigated. As shown in Table 1, the recovery of the sensor for TYR activity was in the range of $94.0-113.0 \%$ with relative standard deviations (RSDs) ranging from $0.52 \%-8.88 \%$. These results demonstrated that the proposed method had a potential application for the practical measurement of TYR activity.

Table 1 Recovery and RSD results of TYR activity in diluted serum samples

\begin{tabular}{lllrl}
\hline Serum sample & $\begin{array}{l}\text { Added } \\
\left(\mathrm{U} \mathrm{mL}^{-1}\right)\end{array}$ & $\begin{array}{l}\text { Measured } \\
\left(\mathrm{U} \mathrm{mL}^{-1}\right)\end{array}$ & Recovery $(\%)$ & $\begin{array}{l}\text { RSD (\%) } \\
(n=3)\end{array}$ \\
\hline 1 & 0.5 & 0.52 & 104.0 & 6.34 \\
2 & 0.5 & 0.47 & 94.0 & 8.88 \\
3 & 1.0 & 1.04 & 104.0 & 6.99 \\
4 & 1.0 & 1.13 & 113.0 & 2.13 \\
5 & 1.5 & 1.55 & 103.3 & 4.39 \\
6 & 2.0 & 2.13 & 106.5 & 1.70 \\
7 & 5.0 & 4.72 & 94.4 & 0.52
\end{tabular}




\section{Conclusions}

In summary, using N-CDs as a simple probe, an easy, sensitive and homogeneous fluorescence strategy for the monitoring of TYR activity was developed. TYR catalyzed the oxidation of DA to $o$-dopaquinone, which effectively quenched the fluorescence of the N-CDs. The use of the proposed assay was shown to be feasible for clinical practice because of the broad linear range and acceptable detection limit of this method for monitoring the TYR activity. Furthermore, by comparing the response results of CDs to different types of $\mathrm{N}$ doping and various levels of amino group content in the TYR catalyzed DA, it was found that the improved degree of $\mathrm{N}$ doping and high amino content were favourable for obtaining a more effective and sensitive assay for the determination of TYR. With the development of $\mathrm{N}$ doped CDs and the increase of some active enzymes through the production/consumption of quinones, it is believed that the design of the surface amino content of fluorescent nanomaterials, such as CDs or other QDs, will be of great help in the development and exploitation of new methods for the determination of TYR.

\section{Conflicts of interest}

There are no conflicts to declare.

\section{Acknowledgements}

The authors gratefully acknowledge the financial support from the Joint Fund for the Innovation of Science and Technology, Fujian province (2017Y9121), the National Science Foundation of Fujian Province (2017J01328), and the Elite Cultivation Program of Health and Family Planning of Fujian Province (2017-ZQN-39, 2017-ZQN-61).

\section{Notes and references}

1 L. H. Stevens, E. Davelaar, R. M. Kolb, E. J. M. Pennings and N. P. M. Smit, Phytochemistry, 1998, 49, 703.

2 R. Freeman, J. Elbaz, R. Gill, M. Zayats and I. Willner, Chem. Eur. J., 2007, 13, 7288.

3 Y. Teng, X. F. Jia, J. Li and E. Wang, Anal. Chem., 2015, 87, 4897.

4 S. Halaouli, M. Asther, J. C. Sigoillot, M. Hamdi and A. Lomascolo, J. Appl. Microbiol., 2006, 100, 219.

5 T. S. Chang, Int. J. Mol. Sci., 2009, 10, 2440.

6 M. N. Zhou, C. P. Guan, F. Q. Lin, W. Xu, L. F. Fu, W. S. Hong, Y. S. Wan and A. Xu, Int. J. Mol. Med., 2011, 27, 725.

$7 \mathrm{H}$. Ando, H. Kondoh, M. Ichihashi and V. J. Hearing, J. Invest. Dermatol., 2007, 127, 751.

8 Y. J. Kim and H. Uyama, Cell. Mol. Life Sci., 2005, 62, 1707. 9 B. Shah and A. Chen, Electrochem. Commun., 2012, 25, 79.

10 G. M. Jiang, X. F. Gu, G. Q. Jiang, T. T. Chen, W. Y. Zhan and S. Tian, Sens. Actuators, B, 2015, 209, 122.
11 J. H. Zhao, X. F. Bao, S. Wang, S. S. Lu, J. Sun and X. R. Yang, Anal. Chem., 2017, 89, 10529.

12 X. F. Wu, L. H. Li, W. Shi, Q. Y. Gong and H. M. Ma, Angew. Chem., Int. Ed., 2016, 55, 14728.

13 J. J. Hu, X. L. Bai, Y. M. Liu and X. Liao, Anal. Chim. Acta, 2017, 995, 99.

14 H. Ao, Z. S. Qian, Y. Y. Zhu, M. Z. Zhao, C. Tang, Y. Y. Huang, H. Feng and A. J. Wang, Biosens. Bioelectron., 2016, 86, 542.

15 J. Y. Sun, H. Mei, S. F. Wang and F. Gao, Anal. Chem., 2016, 88, 7372 .

16 L. J. Chai, J. Zhou, H. Feng, C. Tang, Y. Y. Huang and Z. S. Qian, ACS Appl. Mater. Interfaces, 2015, 7, 23564.

17 F. M. Zhang, L. B. Xu, Q. N. Zhao, Y. Sun, X. H. Wang, P. Y. Ma and D. Q. Song, Sens. Actuators, B, 2018, 256, 1069.

18 X. Yan, H. X. Li, W. S. Zheng and X. G. Su, Anal. Chem., 2015, 87, 8904.

19 F. L. Zu, F. Y. Yan, Z. J. Bai, J. X. Xu, Y. Y. Wang, Y. C. Huang and X. G. Zhou, Microchim. Acta, 2017, 184, 1899.

20 T. Feng, X. Z. Ai, G. H. An, P. P. Yang and Y. L. Zhao, ACS Nano, 2016, 10, 4410.

21 J. Zhou, H. Zhou, J. B. Tang, S. E. Deng, F. Yan, W. J. Li and M. H. Qu, Microchim. Acta, 2017, 184, 343.

22 R. B. Guo, B. Chen, F. L. Li, S. H. Weng, Z. F. Zheng, M. Chen, W. Wu, X. H. Lin and C. Y. Yang, Sens. Actuators, B, 2018, 264, 193.

23 G. C. Zuo, A. M. Xie, X. H. Pan, T. Su, J. J. Li and W. Dong, ACS Appl. Nano Mater., 2018, 1, 2376.

24 G. C. Zuo, A. M. Xie, J. J. Li, T. Su, X. H. Pan and W. Dong, J. Phys. Chem. C, 2017, 121, 26558.

25 F. L. Li, Q. Q. Cai, X. L. Hao, C. F. Zhao, Z. J. Huang, Y. J. Zheng, X. H. Lin and S. H. Weng, RSC Adv., 2019, 9, 12462.

26 J. Y. Huang, F. L. Li, R. B. Guo, Y. Y. Chen, Z. Z. Wang, C. F. Zhao, Y. J. Zheng, S. H. Weng and X. H. Lin, Microchim. Acta, 2018, 185, 260.

27 S. Sun, L. Zhang, K. Jiang, A. G. Wu and H. W. Lin, Chem. Mater., 2016, 28, 8659.

28 G. L. Wang, F. Yuan, T. T. Gu, Y. M. Dong, Q. Wang and W. W. Zhao, Anal. Chem., 2018, 90, 1492.

29 J. Yu, N. Song, Y. K. Zhang, S. X. Zhong, A. J. Wang and J. R. Chen, Sens. Actuators, B, 2015, 214, 29.

30 W. J. Lu, X. J. Gong, M. Nan, Y. Liu, S. M. Shuang and C. Dong, Anal. Chim. Acta, 2015, 898, 116.

31 S. H. Weng, D. Liang, H. Z. Qiu, Z. E. Liu, Z. Lin, Z. F. Zheng, A. L. Liu, W. Chen and X. H. Lin, Sens. Actuators, B, 2015, 221, 7.

32 R. Q. Zhang, S. M. Wang, Y. Yang, Y. L. Deng, D. Li, P. Su and Y. Yang, Anal. Bioanal. Chem., 2018, 410, 3779.

33 W. C. Ye, H. Huang, W. W. Yang, X. Wang, C. L. Ren, Q. S. Hu, Y. M. Li and B. Ren, Analyst, 2017, 142, 3459.

34 G. Y. Liu, J. H. Zhao, S. S. Lu, S. Wang, J. Sun and X. R. Yang, ACS Sens., 2018, 3, 1855.

35 Y. Z. Ding, W. F. Wang, T. Chai, Y. Qiang, Y. P. Shi and J. L. Yang, Talanta, 2019, 197, 113. 